infarction and vasculitis. CSF showed a protein of $98 \mathrm{mg} / \mathrm{dL}$, and PCR evidence of VZVDNA. Heparin and acyclovir were started and 10 days later, aspirin $(50 \mathrm{mg} / \mathrm{day})$ to prevent recurrence of infarction. The paresis improved and at 1-year follow-up, she had minimal reduction in motility of the left arm and leg during sports. MRI showed punctate gliosis and malacia in right semiovale.

A total of 70 published cases of childhood stroke syndrome associated with VZV are reviewed, median age 5 years (range 6 months- 11 years), 43 boys and 28 girls; 10 patients $(14 \%)$ had underlying diseases or prothrombotic conditions (diabetes, AIDS, patent foramen ovale, iron deficiency anemia, protein C deficiency) and $61(86 \%)$ were previously healthy and without risk factors for stroke. Stroke was preccded by chickenpox in $90 \%$ cases and by herpes zoster in 10\%, mainly ophthalmic HZ. In 2 cases, stroke followed vaccination with VZV, and in one at birth secondary to maternal $\mathrm{HZ}$ in the $3^{\text {rd }}$ trimester of pregnancy. Median interval between chickenpox or $\mathrm{HZ}$ and stroke was 18 weeks (range, $0-4$ years). Stroke preceded chickenpox exanthema by 24 hours in 1 case and 2 weeks after viral exposure. Role of treatment is unclear (no treatment in $17 \%$, aspirin in $52 \%$, acyclovir $44 \%$, corticosteroids $40 \%$, heparin $15 \%$ ) and outcome is usually good, with complete recovery in 50\%, including those untreated. (Ciccone S, Faggioli R, Calzolari F, Sartori S, Calderone M, Borgna-Pignatti C. Stroke after varicella-zoster infection. Report of a case and review of the literature. Pediatr Infect Dis J Sept 2010;29(9):864-867). (Respond: Dr Sara Ciccone, Clinica Pediatrica, Universita di Ferrara, 44100 Ferrara, Italy. E-mail: $\underline{\text { saraciccone.unife@ gmail.com). }}$

COMMENT. History of recent chickenpox or $\mathrm{HZ}$ should be considered as a possible cause in a child presenting with ischemic stroke. This report adds support for universal immunization against VZV.

Dr Charles Grose, University of Iowa, who had reviewed all the literature on the complications of varicella infection in children in 1994, reports that stroke was rarely mentioned (Pediatr Infect Dis J 2010;29:868-869). MRI is the reason for the emergence of more case reports of stroke after varicella in the past 2 decades. Stroke after zoster infection is more likely if the location is the eye (zoster ophthalmicus). VZV infects the trigeminal ganglion and other cerebral ganglia, and later reactivates, spreading along sensory nerve fibers to an eye and a cerebral artery, where viral replication causes inflammation and thrombosis. Treatment of zoster ophthalmicus with acyclovir is recommended. Neurological complications of VZV have been underestimated.

\title{
CEREBRAL COMPLICATIONS OF HYPERTENSION
}

Acute and chronic neurological complications of hypertension in patients younger than 21 years were reviewed in the literature, 1980-2010, by researchers at Maimonides Childrens' Hospital, State University and Mount Sinai School of Medicine, New York; and UCL Institute of Child Health, London, UK. Childhood hypertension is underdiagnosed and is defined as an average pressure above the $95^{\text {th }}$ percentile for sex, age, and height on 3 occasions. In a series of 212 pediatric patients with arterial ischemic stroke, systolic BP at immediate follow-up was $>90^{\text {th }}$ percentile in $54 \%$ with symptomatic stroke and in $46 \%$ with cryptogenic stroke. Children with moya-moya disease and stroke were 3-4 times more likely to have hypertension than those with normal vessels. Masked 
hypertension may be an unrecognized risk factor for cerebrovascular disease. In a Chinese study only 4 of 251 patients with stroke had hypertension. Hypertension is an identified factor in stroke with sickle cell disease, but with this exception, there is no definite consensus for an association of hypertension and ischemic stroke in children. Hemorrhagic stroke with hypertension was reported in 15 patients with sickle cell disease, and 6-8\% of hemorrhagic stroke in children were associated with hypertension. Posterior reversible encephalopathy syndrome (PRES) arises with acute severe hypertension and is characterized by headaches, blindness, confusion, and seizures, and evidence of reversible posterior leukoencephalopathy with edema on MRI. The cause is a breakdown of autoregulation and endothelial dysfunction.

Chronic effects of hypertension include cognitive dysfunction affecting attention, learning and memory, reversible with antihypertensive treatment. Hypertension might predispose to endothelial damage and atherosclerosis, as suggested by carotid ultrasound measurements of intima-media thickness, a biomarker of hypertensive vascular damage. Retrospective review of neurological symptoms in 409 children with hypertension aged 7-17 years, and compared with 150 healthy controls, showed $54 \%$ had significantly more complaints of pain, sleep disturbance, fatigue, or lack of concentration. Headache incidence of $42 \%$ before antihypertensive treatment fell to 6-9\% after treatment. (Croix B, Feig DI. Childhood hypertension is not a silent disease. Pediatr Nephrol 2006;21:527-532). Future studies should standardize the definition and monitoring of hypertension in children, investigate transcranial doppler changes correlated with cognitive decline, and investigate the value of MRI in definition of subtle infarcts and small vessel disease. (Sharma M, Kupferman JC, Brosgol Y et al. The effects of hypertension on the paediatric brain: a justifiable concern. Lancet Neurol Sept 2010;9:933-940). (Respond: Prof SG Pavlakis, Maimonides Infants and Childrens' Hospital, Brooklyn, NY 11219. E-mail: spavlakis@maimonidesmed.org).

COMMENT. The authors find pediatric hypertension is underdiagnosed and except for hypertensive encephalopathy, the neurological effects are under-recognized. With the increased incidence of obesity and diabetes type 2 in children, hypertension has become more prevalent. Patients at risk of hypertension should receive more frequent monitoring, so that chronic effects of hypertension on cognition and memory may be avoided.

\section{$\underline{\text { SEIZURE DISORDERS }}$}

\section{INFANTILE SPASMS TREATED WITH THE KETOGENIC DIET}

Researchers at Johns Hopkins Hospital, Baltimore, MD have evaluated the efficacy of the ketogenic diet in the treatment of 104 consecutive infants with infantile spasms (IS) and hypsarrhythmia on EEG. The cohort included 23 of initial patients seen, 13 new-onset IS, and 68 additional patients. The etiology was symptomatic in $74(71 \%)$, and previous therapy had included a mean of 3.6 anticonvulsants, with corticosteroids or vigabatrin in $71 \%$. Diet efficacy was assessed through patient clinic visits at $3,6,9,12$, and 24 months, and by telephone and e-mail contact. Developmental progress was based on parental reports and clinical examination. Follow-up EEG was obtained after 6-12 